\title{
Odontogenic Myxoma. A case report
}

\author{
Dr S Hemavathy ${ }^{1}$, Dr Dandena Vinay Kumar² \\ ${ }^{I}$ (Oral Pathology, GDCRI Bangalore/RGUHS,India) \\ ${ }^{2}$ (Oral Pathology, GDCRI Bangalore/RGUHS,India)
}

\begin{abstract}
Myxomas of the jaws are rare odontogenic neoplasm predominantly found in young adults. It commonly occurs in the second and third decade, and the mandible is involved more commonly than the maxilla. It constitute around 3-6\% of total odontogenic tumours and most of the times it grows without symptoms and presents as a painless swelling. Here we are presenting a case of odontogenic myxomas $(O M)$ of 22 yr. old patient in upper left maxillary region.
\end{abstract}

Key words: Myxoma, Mandible, Maxilla, Odontogenic Myxoma, odontogenic tumor.

\section{Introduction}

Myxomas of the jaws are believed to arise from odontogenic ectomesenchyme as it bears close resemblance to the mesenchymal portion of a developing tooth. It was probably first described by Rudolf Virchow as myxofibroma in 1863.[1] [2] In 1992, WHO defined OM as a locally invasive neoplasm consisting of rounded and angular cells lying in an abundant mucoid stroma. These tumors are rare, benign does not show metastasis but they are locally aggressive involving the maxilla and mandible. The average age for patients with myxomas is 25 to 30 yrs.[3]They most frequently involve the posterior mandible and less commonly the maxilla. These myxomas are symptomless when they are in smaller size and are discovered only during a radiographic examination. Larger lesions are often associated with a painless expansion of the involved bone. Here we are presenting a case of odontogenic myxomas which occurred in upper left maxillary region.

\section{Case report}

A 22 year old patient presented with complaint of painless swelling in left middle third of the face since six months. His past medical history was of no relevance and general physical status was good. Extra oral examination revealed swelling in middle third of the face which extends from ala of the nose to the tragus of ear which is localised and non-tender(Fig 1).On intra oral examination there is slight obliteration of buccal vestibule in the region of 22 to 26 with negative aspiration (Fig2). OPG reveals diffused radiolucency which extends from 22 to 27 (Fig 3). Occlusal radiograph is showing fine septae in the radiolucent lesion along the palatal aspect (Fig 4). IOPA shows minimal resorption of roots was seen with respect to 24 to 27(Fig 5). Incisional biopsy of the lesion was done and sent for histopathological examination. On microscopic examination it showed loosely arranged angular, spindle and comma shaped cells resembling dental papillary cells in a delicate myxoid stroma suggestive of odontogenic myxoma.

The tumor was surgically excised along with the removal of tooth involved under general anesthesia. Gross examination of the excised specimen shows white gelatinous appearance (Fig 6). On microscopic examination it shows similar features of incisional biopsy (Fig7,8 and 9). Patient recovery was eventful and has been on regular follow up for the past three months.

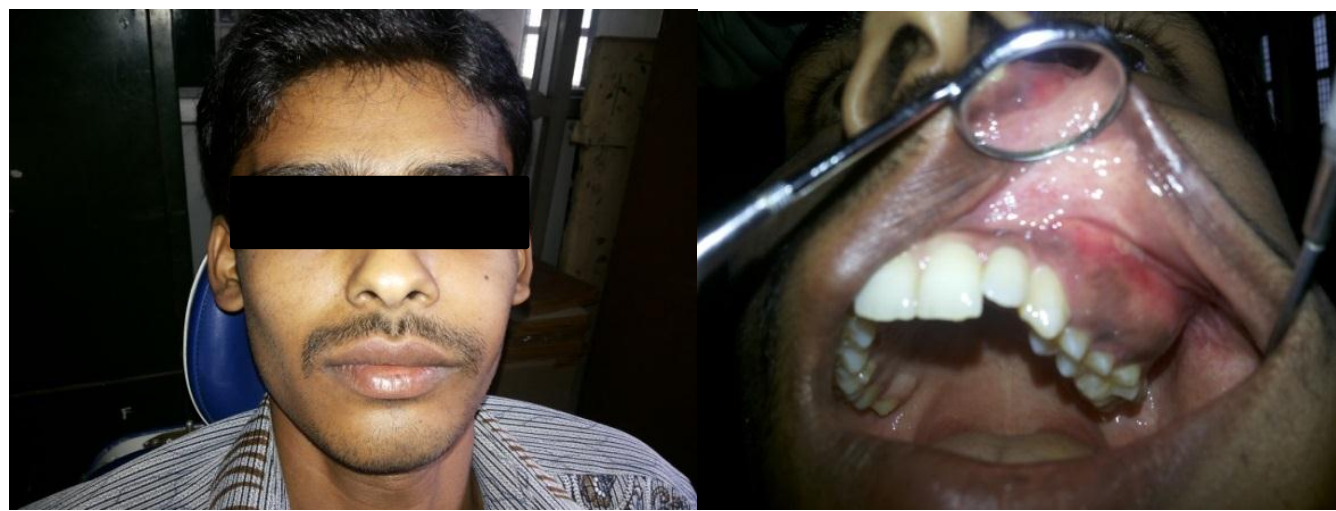

Fig lextra oral photograph view showing swelling left side of the face.

Fig 2 (right side).intraoral view showing vestibular obliteration extending from 23 to 26. 


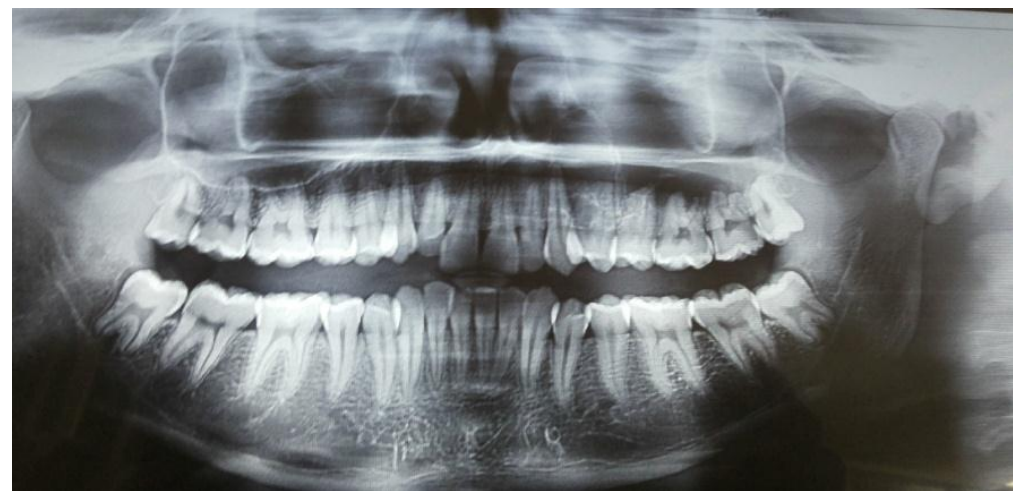

Fig 3.OPG showing ill defined radiolucency extends from 23 to 27 region

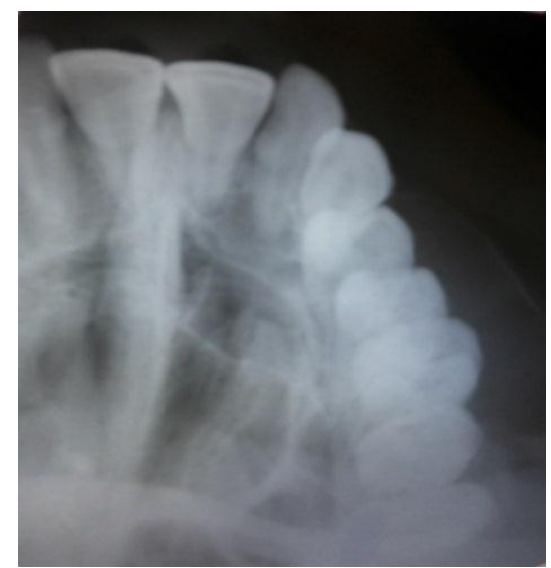

Fig 4.Occlusal radiograph showing fine septae in the radiolucent lesion along the palatal aspect

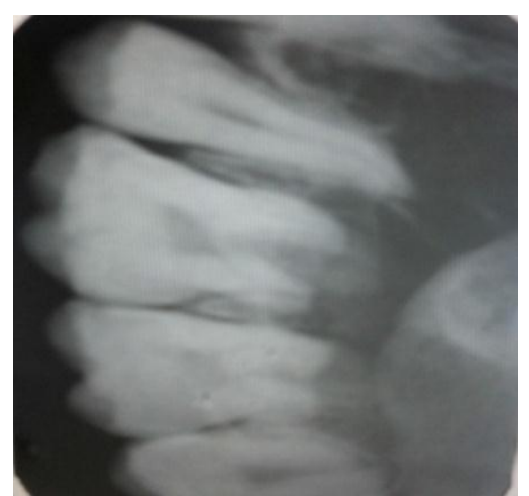

Fig 5.IOPA showing periapical radiolucency showing mild resorption of roots extending from 24 to 27

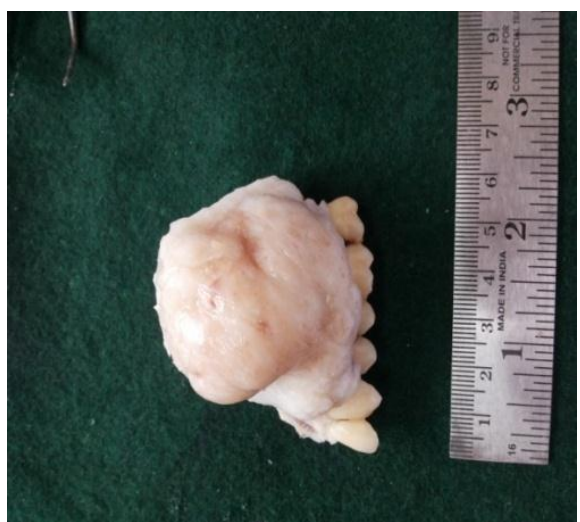

Fig6. Excisional biopsy showing odontogenic myxoma which is white gelatinous mass measuring around $6 \times 5 \mathrm{~cm}$. 


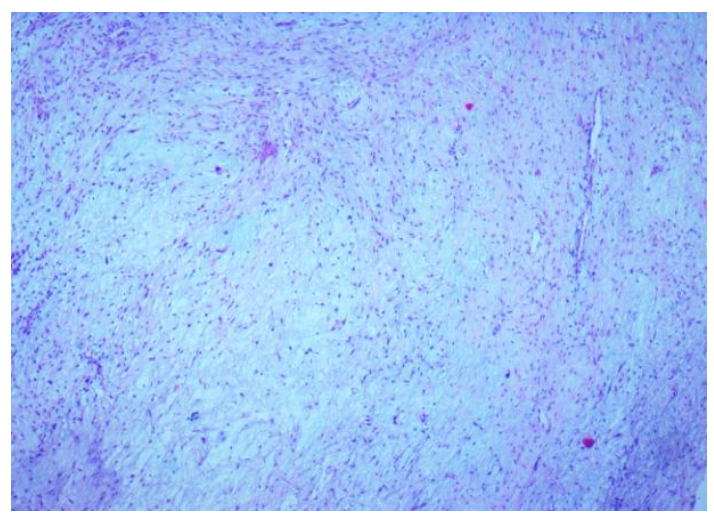

Fig 7.Showing odontogenic myxoma which is highly cellular in a myxoid stroma(4X).

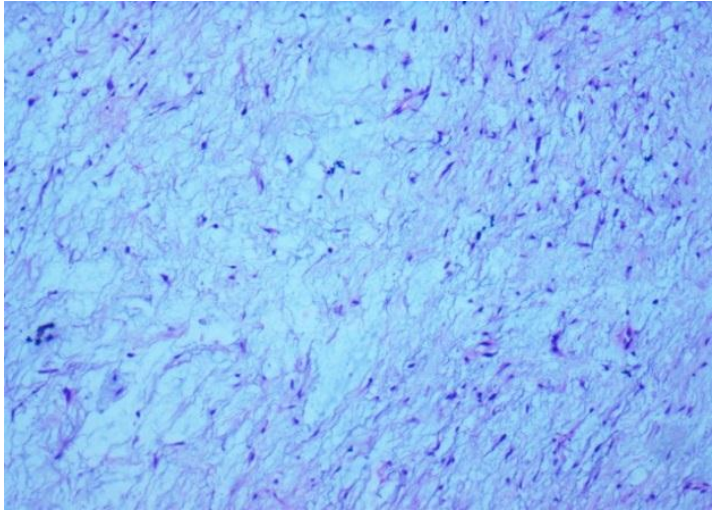

Fig 8. Showing dental papillary, spindle shaped cells in a myxoid stroma.(10X)Figure 8. Showing dental papillary, spindle shaped cells in a myxoid stroma.(10X)

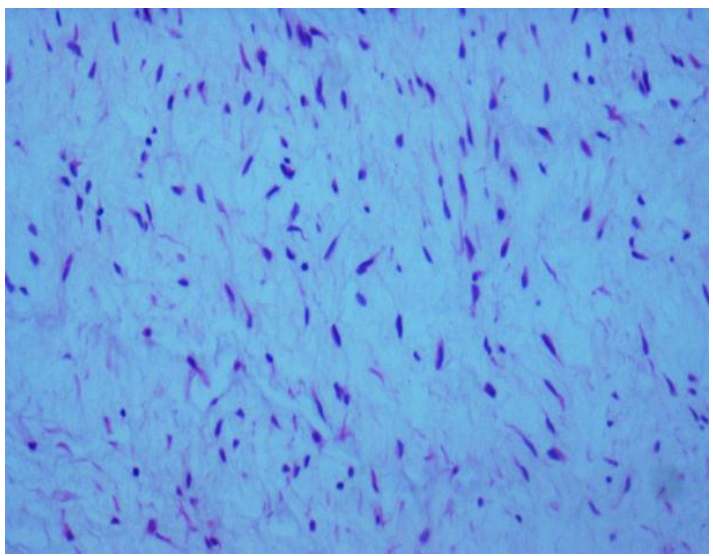

Fig9. Showing odontogenic myxoma (40X).

\section{Discussion}

Odontogenic myxomas $(\mathrm{OM})$ are rare tumors derived from embryonic mesenchymal elements of dental anlage. World Health Organization has classified OM as a benign tumor of ectomesenchymal origin with or without odontogenic epithelium. It appears to originate from the dental papilla, follicle or periodontal ligament. Myxomas are non-encapsulated benign locally aggressive tumors which may occur in both the soft tissues and bone. In 1863 Virchow was the first man to describe about myxomas. He described that these tumors resemble the mucinous substance of the umbilical cord. These tumors are most frequently seen in heart muscle. Myxomas of the mandible and maxilla are very rare. In 1947, Thoma and Goldman separated myxomas of the mandible and maxilla from other myxomas. In 1948, Stout redefined the histological criteria for myxomas as benign neoplasms of mesenchymal origin.[4]

Clinically these tumors most commonly seen in young adults but may occur over a wide age group. The average age for patients with myxomas is second and third decade of life.[5] There is no sex predilection. The tumor may found in any area of the jaws but the mandible is most common area than maxilla. Smaller 
lesions may be asymptomatic and are discovered only during a radio graphic examination. Larger lesions are often associated with a painless expansion of the involved bone.[4][5][6]

Radiologically OM may appear as a unilocular or multilocular radiolucency with either honeycomb, or soap-bubble pattern. In most of the cases due to local aggressive nature there will be cortical expansion and tooth displacement. Approximately half of the cases showed root resorption. The radiolucency may have clearly defined borders or poorly defined diffuse borders. [5]

On gross examination myxoma appears as a mass of characteristically mucoid, white gelatinous slimy material and sometimes covered by eggshell patches of bone.[3]

Microscopically, odontogenic myxoma is a benign neoplasm without encapsulation and exhibits a spectrum of fibrous connective tissue stroma from myxoid to densely hyalinized and from relatively acellular to cellular nature. Majority of the tumor shows loosely arranged stellate or spindle shaped cells resembling dental papillary cells in a delicate myxoid stroma with few collagen fibers. [4] And in some cases cells were active with granular cytoplasm, dense diffuse collection of fibrils and fibroblasts with small inconspicuous strands of odontogenic epithelium. Due to dense collection of fibrils and fibroblasts it was called odontogenic fibromyxoma.[7]

Treatment planning for myxomas varies depending upon the extension of the lesion. Small tumors are generally treated by curettage, but careful periodic reevaluation is necessary for at least 5 years is necessary because of high recurrence rate of approximately $25 \%$. For larger lesions more extensive resection may be required as myxomas are not encapsulated and tend to infiltrate the surrounding bone. Complete removal of a large tumor by curettage is often difficult to accomplish, and lesions of the posterior maxilla, in particular, should be treated more aggressively in most instances. But some surgeons recommend simple enucleation and curettage for fibrotic lesions, and in pediatric cases, because of the concern for facial disfigurement and potential interference with growth centers. In spite of local recurrences, the overall prognosis is good, and metastases do not occur.[3][7][8]

\section{Conclusion}

It is difficult to diagnose OM based on clinical and radiographic finding. Diagnosis can be made by biopsy. Differential diagnosis has great importance for all tumors involving the maxillo-facial region. Recurrence rates are high and a long follow-up period over years is essential after treatment for patients with these tumors. Due to aggressive behavior of these tumors regular follow up is absolutely necessary in the cases of odontogenic myxomas.

\section{References}

[1] Vasudevan V et al, Odontogenic myxaoma of the maxilla. International journal of clinical paediatric dentistry, 4(3),2011,264-68

[2] Meghanand T, Anjali S, Madhusudan A.Maxillary Odontogenic Myxoma A Rarity.International Journal of Oral \& Maxillofacial Pathology,2(3),2011,32-35.

[3] CD Mounesh Kumar, KV Suresh, Praveen Reddy B, KV Umashankar.Odontogenic Fibromyxoma of Maxilla: A Case Report.Journal of Krishna Institute of Medical Sciences University 2(1),2013, 120-24.

[4] Mustafa K, Selçuk Y.Odontogenic myxomas of the maxilla,Clinics and Practice, 1,2011,304-305.

[5] Shafer, Hine, Levy.Text book of Oral Pathology. $5^{\text {th }}$ edition, Elsevier New Delhi (2006).

[6] Neville BW, Damm DD, Allen CM, Bouquot JE. Oral and Maxillofacial Pathology. 2 $2^{\text {nd }}$ edition, Elsevier New Delhi (2002).

[7] Elison NM, Matthias AW, Edda V, David N, Paul J W. Odontogenic myxoma: a clinicopathological study of 33 cases.Int J Oral MaxillofacSurg, 33,2004,333-337.

[8] Dorrit WN, Dan G, Badri A.Childhood odontogenic myxoma: report of two cases.The American Academy of Pediatric Dentistry, $7(2), 1985,140-44$. 